\title{
Combined Semianalytical and Numerical Static Plate Analysis. Part 2: Resultant Multipoint Boundary Problem
}

\author{
Oleg Negrozov ${ }^{1,2}$, Pavel Akimov ${ }^{1,2, *}$, and Marina Mozgaleva ${ }^{1}$ \\ ${ }^{1}$ Moscow State University of Civil Engineering, Yaroslavskoe Shosse, 26, Moscow, 129337, Russia \\ ${ }^{2}$ Russian Academy of Architecture and Construction Sciences; 24-1, ul. Bolshaya Dmitrovka, \\ Moscow, 107031, Russia
}

\begin{abstract}
The distinctive paper is devoted to solution of multipoint boundary problem of plate analysis (Kirchhoff model) based on combined application of finite element method (FEM) and discrete-continual finite element method (DCFEM). As is known the Kirchhoff-Love theory of plates is a two-dimensional mathematical model that is normally used to determine the stresses and deformations in thin plates subjected to forces and moments. The given domain, occupied by considering structure, is embordered by extended one. The field of application of DCFEM comprises fragments of structure (subdomains) with regular (constant or piecewise constant) physical and geometrical parameters in some dimension ("basic" dimension). DCFEM presupposes finite element mesh approximation for non-basic dimension of extended domain while in the basic dimension problem remains continual. FEM is used for approximation of all other subdomains (it is convenient to solve plate bending problems in terms of displacements). Coupled multilevel approximation model for extended domain and resultant multipoint boundary problem are constructed. Brief information about software systems and verification samples are presented as well.
\end{abstract}

\section{Boundary conditions}

Boundary conditions at section $x_{2}=x_{2,1}^{b}$ (hinged edge) has the form ( $4 N_{1}$ equations):

$$
\begin{gathered}
y_{1}^{(1, i)}\left(x_{2,1}^{b}+0\right)=0, \quad i=1,2, \ldots, N_{1} ; \\
z_{1}^{(1, i)}\left(x_{2, k}^{b}+0\right)=0, \quad i=1,2, \ldots, N_{1} ; \\
y_{3}^{(1, i)}\left(x_{2, k}^{b}+0\right)=0, \quad i=1,2, \ldots, N_{1} ;
\end{gathered}
$$

\footnotetext{
* Corresponding author: pavel.akimov@gmail.com
} 


$$
z_{3}^{(1, i)}\left(x_{2, k}^{b}+0\right)=0, \quad i=1,2, \ldots, N_{1}
$$

Equations (1)-(4) can be rewritten in matrix form:

$$
B_{1}^{+} \bar{Y}_{1}\left(x_{2,1}^{b}+0\right)=\bar{g}_{1}^{+},
$$

where $B_{1}^{+}$is matrix of boundary conditions of size $4 N_{1} \times 8 N_{1}$, which can be constructed in accordance with algorithm presented at Table $1 ; \bar{g}_{1}^{+}$is the zero vector of order $4 N_{1}$,

$$
\bar{g}_{1}^{+}=0
$$

Table 1. Algorithm of construction of matrix $B_{1}^{+}$(all other elements of matrix $B_{1}^{+}$are equal to zero).

\begin{tabular}{|c|c|c|}
\hline Numbers (indexes) of elements & Element value & Corresponding boundary condition \\
\hline$(i, 2 i-1), i=1,2, \ldots, N_{1}$ & 1 & Equations (1) \\
\hline$\left(N_{1}+i, 2 i\right), i=1,2, \ldots, N_{1}$ & 1 & Equations (2) \\
\hline$\left(2 N_{1}+i, 4 N_{1}+2 i-1\right), i=1,2, \ldots, N_{1}$ & 1 & Equations (3) \\
\hline$\left(3 N_{1}+i, 4 N_{1}+2 i\right), i=1,2, \ldots, N_{1}$ & 1 & Equations (4) \\
\hline
\end{tabular}

After substitution of formula (11) from the first part of this paper into (5) it can be obtained that

$$
Q_{1} \bar{C}_{1}=\bar{G}_{1}
$$

where $Q_{1}$ is the matrix of size $4 N_{1} \times 8 N_{1} ; \bar{G}_{1}$ is the vector of order $4 N_{1}$,

$$
Q_{1}=B_{1}^{+} E_{1}\left(x_{2,1}^{b}+0\right), \quad \bar{G}_{1}=\bar{g}_{1}^{+}-B_{1}^{+} \bar{S}_{1}\left(x_{2,1}^{b}+0\right) \text {. }
$$

Boundary conditions at section $x_{2}=x_{2,2}^{b}$ (perfect contact, Fig. 1) has the form $\left(4 N_{1}\right.$ equations):

$$
\begin{gathered}
y_{1}^{(1, i)}\left(x_{2,2}^{b}-0\right)=y_{1}^{(2, i, j)}, i=1,2, \ldots, N_{1}, \quad j=1 ; \\
z_{1}^{(1, i)}\left(x_{2,2}^{b}-0\right)=z_{1}^{(2, i, j)}, i=1,2, \ldots, N_{1}, j=1 ; \\
y_{2}^{(1, i)}\left(x_{2,2}^{b}-0\right)=y_{2}^{(2, i, j)}, i=1,2, \ldots, N_{1}, j=1 ; \\
z_{2}^{(1, i)}\left(x_{2,2}^{b}-0\right)=\left[\partial_{1} y_{2}\right]^{(2, i, j)}, i=1,2, \ldots, N_{1}, \quad j=1 ; \\
M_{2}^{(1, i)}\left(x_{2,2}^{b}-0\right)=M_{2}^{(2, i, j)}, i=1,2, \ldots, N_{1}, \quad j=1 ;
\end{gathered}
$$




$$
\begin{gathered}
{\left[\partial_{1} M_{2}\right]^{(1, i)}\left(x_{2,2}^{b}-0\right)=\left[\partial_{1} M_{2}\right]^{(2, i, j)}, i=1,2, \ldots, N_{1}, \quad j=1 ;} \\
V_{2}^{(1, i)}\left(x_{2,2}^{b}-0\right)=V_{2}^{(2, i, j)}, i=1,2, \ldots, N_{1}, \quad j=1 ; \\
{\left[\partial_{1} V_{2}\right]^{(1, i)}\left(x_{2,2}^{b}-0\right)=\left[\partial_{1} V_{2}\right]^{(2, i, j)}, i=1,2, \ldots, N_{1}, \quad j=1 ;}
\end{gathered}
$$

where $M_{2}^{(1, i)}\left(x_{2}\right), V_{2}^{(1, i)}\left(x_{2}\right)$ and $\left[\partial_{1} M_{2}\right]^{(1, i)}\left(x_{2}\right),\left[\partial_{1} V_{2}\right]^{(1, i)}\left(x_{2}\right)$ are nodal functions (after corresponding averaging) of bending moment $M_{2}$, adjusted shear force $V_{2}$ and corresponding derivatives with respect to $x_{1}\left(\partial_{1} M_{2}, \partial_{1} V_{2}\right)$ for discrete-continual finite element $(1, i) ; M_{2}^{(2, i, j)}, V_{2}^{(2, i, j)}$ and $\left[\partial_{1} M_{2}\right]^{(2, i, j)},\left[\partial_{1} V_{2}\right]^{(2, i, j)}$ are nodal bending moment $M_{2}$, adjusted shear force $V_{2}$ and corresponding derivatives with respect to $x_{1}\left(\partial_{1} M_{2}, \partial_{1} V_{2}\right)$ for finite element $(2, i, j) ; j=1$.

Equations (9)-(16) can be rewritten in matrix form:

$$
B_{2}^{-} \bar{U}_{1}\left(x_{3,2}^{b}-0\right)=B_{2}^{+} \bar{U}_{2},
$$

where $B_{2}^{-}$is matrix of boundary conditions of size $8 N_{1} \times 8 N_{1}$, which can be constructed in accordance with so-called method of basis variations $[1,26] ; B_{2}^{+}$is matrix of boundary conditions of size $8 N_{1} \times 4 N_{1} N_{2}$, which can be constructed in accordance with method of basis variations $[1,26]$.

After substitution of (11) into (17) it can be obtained that

$$
Q_{2,1} \bar{C}_{1}+Q_{2,2} \bar{Y}_{2}=\bar{G}_{2},
$$

where $Q_{2,1}$ and $Q_{2,2}$ are matrices with sizes $8 N_{1} \times 4 N_{1}$ and $8 N_{1} \times 4 N_{1} N_{2} ; \bar{G}_{2}$ is the vector of order $8 N_{1}$,

$$
Q_{2,1}=B_{2}^{-} E_{1}\left(x_{2,2}^{b}-0\right) ; \quad Q_{2,2}=-B_{2}^{+} ; \quad \bar{G}_{2}=-B_{2}^{-} \bar{S}_{1}\left(x_{2,2}^{b}-0\right) .
$$

Boundary conditions at section $x_{2}=x_{2,3}^{b}$ (hinged edge, Figure 1 from the first part of this paper) has the form ( $3 N_{1} N_{2}$ equations):

$$
\begin{gathered}
y_{1}^{(2, i, j)}=0, \quad i=1,2, \ldots, N_{1}, \quad j=N_{2} ; \\
z_{1}^{(2, i, j)}=0, \quad i=1,2, \ldots, N_{1}, \quad j=N_{2} ; \\
{\left[\partial_{2}^{2} y_{1}\right]^{(2, i, j)}=0, i=1,2, \ldots, N_{1}, \quad j=N_{2} ;} \\
{\left[\partial_{1} \partial_{2}^{2} y_{1}\right]^{(2, i, j)}=0, i=1,2, \ldots, N_{1}, \quad j=N_{2} .}
\end{gathered}
$$

Equations (20)-(23) can be rewritten in matrix form:

$$
B_{3}^{-} \bar{Y}_{2}=\bar{g}_{3}^{-},
$$


where $B_{3}^{-}$is matrix of boundary conditions of size $4 N_{1} \times 4 N_{1} N_{2}$, which can be constructed in accordance with method of basis variations $[1,26] ; \bar{g}_{3}^{-}$is the zero vector of order $4 N_{1}$,

$$
\bar{g}_{3}^{-}=0 \text {. }
$$

Thus, the total number of equation is equal to $4 N_{1} N_{2}+8 N_{1}$. Corresponding coupled system of $4 N_{1} N_{2}+8 N_{1}$ linear algebraic equations with $4 N_{1} N_{2}+8 N_{1}$ unknowns has the following form:

$$
\left[\begin{array}{cc}
Q_{1} & 0 \\
Q_{2,1} & Q_{2,2} \\
0 & \widetilde{K}_{2} \\
0 & B_{3}^{-}
\end{array}\right]\left[\begin{array}{l}
\bar{C}_{1} \\
\bar{U}_{2}
\end{array}\right]=\left[\begin{array}{c}
\bar{G}_{1} \\
\bar{G}_{2} \\
\widetilde{\widetilde{R}}_{2} \\
\bar{g}_{3}^{-}
\end{array}\right] .
$$

It should be noted that boundary conditions (24) can be taken into account automatically within construction of global stiffness matrix and global right-side vector corresponding to subdomain $\omega_{2}$ [2]. Then we get (instead of (26)):

$$
\left[\begin{array}{cc}
Q_{1} & 0 \\
Q_{2,1} & Q_{2,2} \\
0 & \widetilde{\widetilde{K}}_{2}
\end{array}\right]\left[\begin{array}{c}
\bar{C}_{1} \\
\bar{U}_{2}
\end{array}\right]=\left[\begin{array}{c}
\bar{G}_{1} \\
\bar{G}_{2} \\
\overline{\widetilde{R}}_{2}
\end{array}\right],
$$

where $\widetilde{\widetilde{K}}_{2}$ is corresponding reduced global stiffness matrix of size $\left[4 N_{1}\left(N_{2}-1\right)\right] \times\left[4 N_{1} N_{2}\right]$; $\overline{\widetilde{R}}_{2}$ is corresponding reduced global right-side vector of order $4 N_{1}\left(N_{2}-1\right)$.

Strain and stress components are computed according to well-known formulas after solving of system (27).

\section{About multipoint boundary problem}

As is known two-point boundary problem is a special case of multipoint boundary problem. Generally, the formulation of considering multipoint boundary problem includes three main components: a description of the domain occupied by the structure and the corresponding subdomains; description of the conditions inside the domain and inside subdomains; description of boundary conditions (for boundaries of domain and boundaries between subdomains).

We have to adjust notation system by analogy with $[3-5,15,16,20]$ for multipoint boundary problem. General principles of domain approximation have been already considered. Algorithm of numbering of subdomains is presented in [3-5,20]. Numbering of finite elements, discrete-continual finite elements and construction of multilevel approximation model for domain are carried out by analogy with [3-5,15,16,20].

In practical applications the following variants of boundary conditions (interface conditions) between subdomains (2) are most often encountered: interface "discretecontinual model - discrete model", "internal" boundary condition of the type "perfect contact"; interface "discrete model - discrete-continual model", "internal" boundary condition of the type "perfect contact"; interface "discrete-continual model - discretecontinual model", "internal" boundary condition of the type "perfect contact"; interface 
"discrete model - discrete model", "internal" boundary condition of the type "perfect contact"; interface "external boundary - discrete model", boundary condition of the type "hinged support"; interface "external boundary - discrete model", boundary condition of the type "free edge"; interface "external boundary - discrete-continual model", boundary condition of the type "hinged support"; interface "external boundary - discrete-continual model", boundary condition of the type "free edge"; interface "discrete model - external boundary", boundary condition of the type "hinged support"; interface "discrete model external boundary", boundary condition of the type "free edge"; interface "discretecontinual model - external boundary", boundary condition of the type "hinged support"; interface "discrete-continual model - external boundary, boundary condition of the type "free edge". Of course, other variants of interface are possible, but similarly, somehow, in one way or another, as a rule, these variants are reduced to some combinations of the above-mentioned twelve [20].

It should be noted, that construction of corresponding matrices of boundary conditions for various variants can be done by analogy with $[3-5,15,16,20]$.

\section{Software system and verification samples. Results and Discussion}

All methods and algorithms considered in this paper have been realized in software system. The main purpose of Analysis system CSASA2DPL (DCFEM + FEM) is semianalytical plate analysis (Kirchhoff plates [10,14,19,21-23]), based on combined application of FEM and DCFEM. Programming environment is Microsoft Visual Studio 2013 Community and Intel Parallel Studio 2017XE (Fortran programming language [13]) with Intel MKL Library. Software system is designed for Microsoft Windows 8.1/10.

Verification samples of structural analysis are presented in [20]. ANSYS Mechanical 15.0 (FEM $[9,24,25]$ ) was used for verification purposes [6-8,11,12,17,18]. We should note that the results of analysis obtained by the ANSYS Mechanical and CSASA2DPL generally agree well with each other. Besides, it is necessary to note that DCFEM is more effective in the most critical, vital, potentially dangerous areas of plate in terms of fracture (areas of the so-called edge effects), where some components of solution are rapidly changing functions and their rate of change in many cases can't be adequately taken into account by the standard FEM.

\section{Conclusion}

Thus, combined semianalytical and numerical static plate analysis based on finite element method (FEM) and discrete-continual finite element method (DCFEM) are presented. Scope of DCFEM includes fragments of considering plate with (piecewise) invariability of physical and geometrical parameters in one dimension (so-called "basic" dimension (direction)). DCFEM presupposes finite element mesh approximation for non-basic dimension of extended domain while in the basic dimension problem remains continual and corresponding analytical solution is constructed. This analytical solution along basic dimension is apparently preferable in all aspects for qualitative analysis of calculation data. Hence it appears that in this context semianalytical analysis considering in this paper is peculiarly relevant. Generally, semianalytical formulations are contemporary mathematical models which currently becoming available for computer realization.

The Reported study was Funded by Government Program of the Russian Federation "Development of science and technology" (2013-2020) within Program of Fundamental Researches of Ministry of 
Construction, Housing and Utilities of the Russian Federation and Russian Academy of Architecture and Construction Sciences, the Research Projects 7.1.1, 7.1.2, 7.4.2, 7.4.3, 7.4.17”.

\section{References}

1. P.A. Akimov, Applied Mechanics and Materials 204-208, 4502-4505 (2012)

2. P.A. Akimov, M.L. Mozgaleva, Applied Mechanics and Materials 580-583, 2898-2902 (2014)

3. P.A. Akimov, O.A. Negrozov, Procedia Engineering 153, 8-15 (2016)

4. P.A. Akimov, O.A. Negrozov, Procedia Engineering 153, 16-23 (2016)

5. P.A. Akimov, O.A. Negrozov, Procedia Engineering 153, 24-31 (2016)

6. E.M. Alawadhi, Finite Element Simulations Using ANSYS (CRC Press, 2009)

7. E.J. Barbero, Finite Element Analysis of Composite Materials Using ANSYS (CRC Press, 2013)

8. K.-J. Bathe, Finite Element Procedures (Prentice Hall Inc., 1996)

9. A. Belostotsky, T. Kaytukov, I. Afanasyeva, J. Grosel, MATEC Web Conf. 2017 116, 00016 (2017)

10. U. Bohinc, B. Brank, A. Ibrahimbegovics, Computer Methods in Applied Mechanics and Engineering 269, 415-436 (2014)

11. E.H. Dill, The Finite Element Method for Mechanics of Solids with ANSYS Applitions (CRC Press, 2011)

12. P. Hansbo, M.G. Larson, Computer Methods in Applied Mechanics and Engineering 200(47-48), 3289-3295 (2011)

13. R.J. Hanson, T. Hopkins, Numerical Computing with Modern Fortran (Applied Mathematics) (SIAM-Society for Industrial and Applied Mathematics, 2013)

14. L. Leonetti, M. Mazza, M. Aristodemo, Engineering Analysis with Boundary Elements 33(1), 1-11 (2009)

15. L. Lyakhovich, O. Negrozov, MATEC Web of Conferences 117, 00109 (2017)

16. L. Lyakhovich, O. Negrozov, MATEC Web of Conferences 117, 00110 (2017)

17. E. Madenci, I. Guven, The Finite Element Method and Applications in Engineering Using ANSYS (Springer, 2015)

18. S. Moaveni, Finite Element Analysis: Theory and Application with ANSYS (Prentice Hall, 2014)

19. Gh. Moraru, Engineering Analysis with Boundary Elements 30(5), 382-390 (2006)

20. O.A. Negrozov, P.A. Akimov, I.Yu. Lantsova, Procedia Engineering 153, 926-932 (2016)

21. G.C. Tsiatas, International Journal of Solids and Structures 46(13), 2757-2764 (2009)

22. K. Vijayakumar, Mechanics Research Communications 38(3), 211-213 (2011)

23. M. Weise, Applied Numerical Mathematics 125, 10-22 (2018)

24. O.C. Zienkiewicz, R.L. Taylor, D.D. Fox, The Finite Element Method for Solid and Structural Mechanics (Butterworth-Heinemann, 2013)

25. O.C. Zienkiewicz, R.L. Taylor, J.Z. Zhu, The Finite Element Method: Its Basis and Fundamentals (Butterworth-Heinemann, 2005)

26. A.B. Zolotov, P.A. Akimov, Proceedings of the International Symposium LSCE 2002 organized by Polish Chapter of IASS (Warsaw, Poland, 2002) 